\title{
Isolated Multifocal Sclerosing Thyroiditis: Case Report
}

\author{
Annalisa Paliotta, Giuseppe Pedullà , Daniele Crocetti, Giuseppe Cavallaro, \\ Giuseppe D’Ermo, Andrea Polistena, Giorgio De Toma \\ Department of Surgery “Pietro Valdoni”, Policlinico Umberto I, University of Rome "Sapienza”, Rome, Italy \\ Email: *pan_gan@hotmail.com
}

Received January 15, 2013; revised February 18, 2013; accepted February 26, 2013

\begin{abstract}
Multifocal fibrosclerosis is a very rare fibroproliferative syndrome involving multiple organ systems. In our report we present the case of multifocal sclerosing thyroiditis characterized by multiple fibroelastic foci similar to breast "radial scar", which can be misdiagnosed as multifocal papillary carcinoma. The diagnosis of multifocal sclerosing thyroiditis, in according with Armed Forces Institute of Pathology (AFIP) textbook on thyroid tumors, was made only after histological and himmunohistochemical examination. We consider the multifocal sclerosing thyroiditis as the first stage of multifocal IgG4-related sclerosing disease for the presence of IgG4 within fibrosclerotic tissue of thyroid. Total body CT-scan and plamatic IgG4 levels must be investigated in order to exclude or confirm the presence of systemic disease.
\end{abstract}

Keywords: Multifocal Sclerosing Thyroiditis; Surgery; IgG4-Related Sclerosing

\section{Introduction}

Multifocal fibrosclerosis, first described by Ormond in 1948 [1] and after by Barrett in 1958 [2] is a very rare fibroproliferative syndrome involving multiple organ systems [3]. Sclerosing cholangitis, retroperitoneal fibrosis, Riedel's Thyroiditis, fibrotic pseudotumor of the orbit and fibrosis of salivary glands have been described and seem to be different clinical manifestations of the same syndrome [3-5]. Multifocal sclerosing thyroiditis, a separate entity from Riedel's thyroiditis, as described by Rosai in 1992, is characterized by multiple fibroelastic foci similar to breast "radial scar", which can be misdiagnosed as multifocal papillary carcinoma. This thyroiditis has now recognized to be an expression of multifocal fibrosclerosis [6].

The Authors describe a case of isolated multifocal sclerosing thyroiditis with no evidence of multiorganic involvement.

\section{Case Report}

A 44-year-old female patient was admitted to our institution for a multinodular normofunctioning goiter, presenting, at ultrasound examination, multiple bilateral thyroid nodules having hypervascularization. Thyroid hormone levels were normal, as it was for laboratory findings. No history of hypertension, cardiovascular or pulmonary disease, no diabetes or malignancies were reported.

In March 2010, the patient underwent complete thy-

${ }^{*}$ Corresponding author. roidectomy, and after an uneventful course, she was discharged after 48 hours.

Histology revealed the presence of multiple, disseminated fibrosclerosis foci, with massive presence of lymphocytes and plasm cells. Due to the presence of nuclear atypies, anti-HBME-1 immunoreaction was performed, to exclude the presence of papillary microcarcinoma. Immunohistochemistry showed intense reaction to IgG4 and IgA antibodies within lymphocytes infiltration and within fibrosclerotic tissue (Figures 1-4). So diagnosis of multifocal sclerosing thyroiditis was given, accordin to Rosai's classification [6].

In order to identify the presence of multifocal fibro-

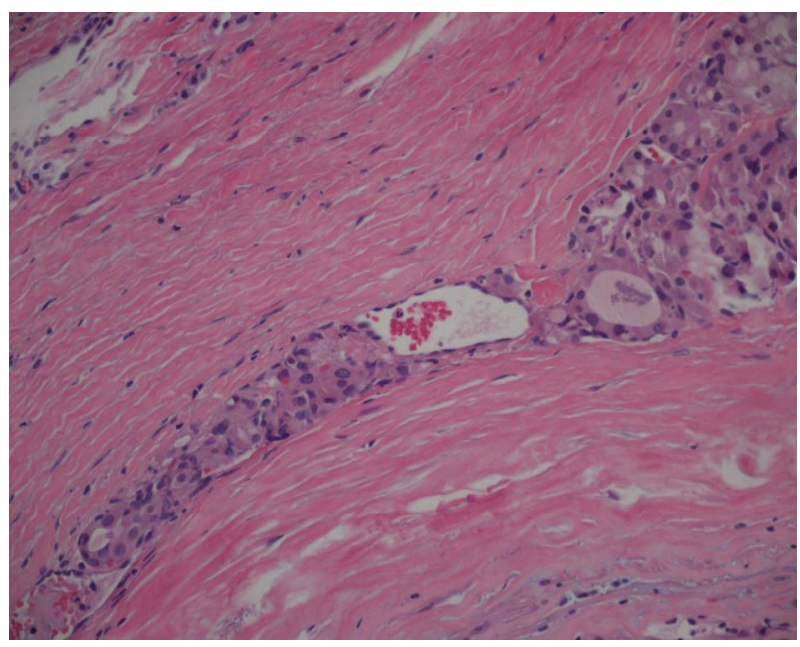

Figure 1. Blood vassel inside the capsule. 


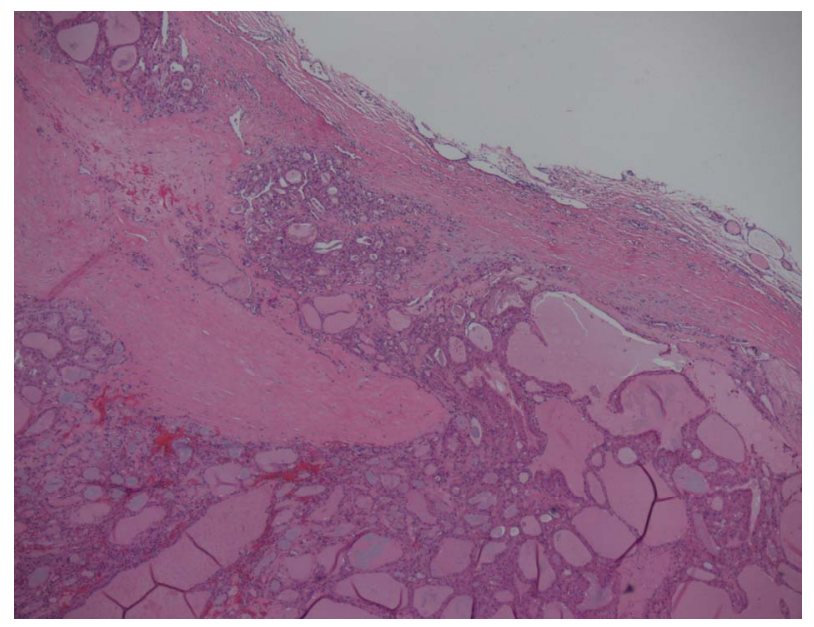

Figure 2. Capsular invasion.

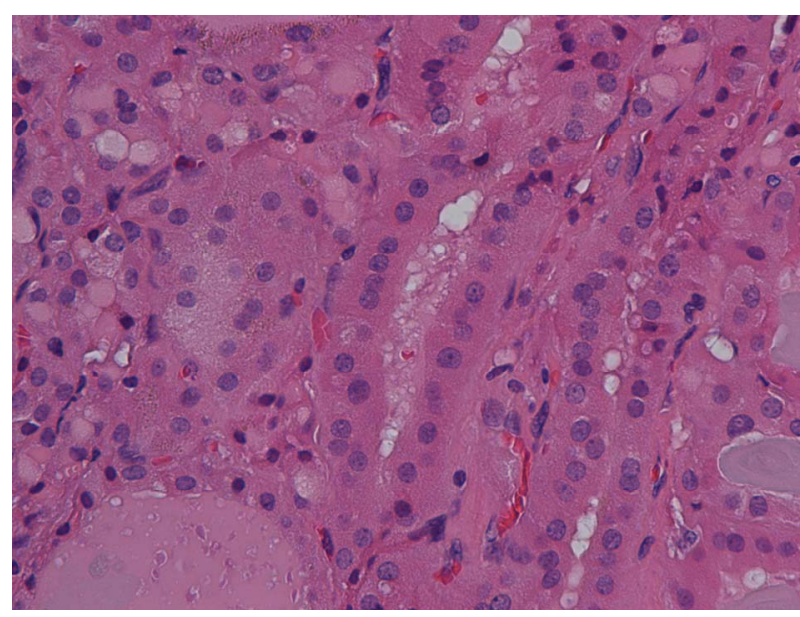

Figure 3. Oxyphilic character of the proliferating elements.

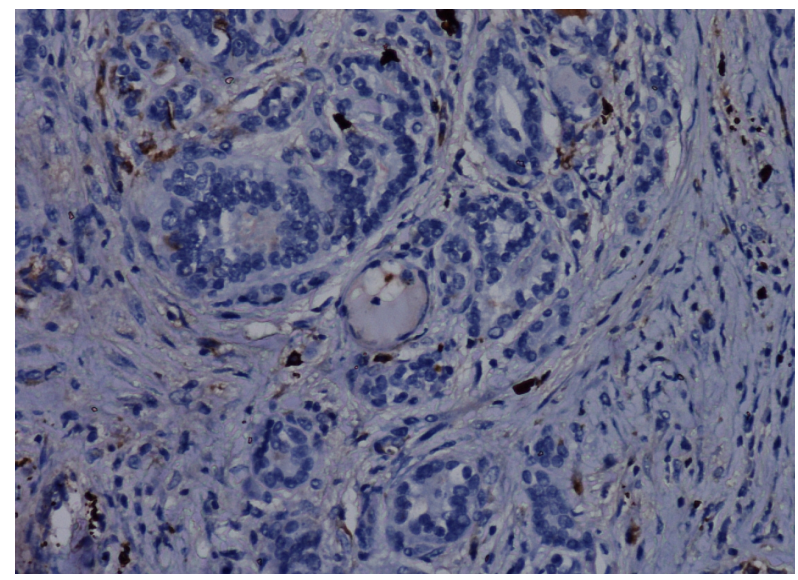

Figure 4. Presence of iga and lymphoid elements at the interface between fibrous proliferation and follicles.

sclerosis, the patient was submitted to total body CT-scan that excluded multiorganic involvement. Furthermore, plasmatic IgG4 levels and anti-Scl70 antibodies were within the normal range. These findings definitely ex- cluded the presence of multifocal fibrosclerosis.

\section{Discussion}

Multifocal fibrosclerosis is a rare syndrome of unknown etiology characterized by fibrosis involving multiple organ systems [2,3]. Retroperitoneal fibrosis, Riedel's thyroiditis, orbital pseudotumor, mediastinal fibrosis and scleroting cholangitis have been reported to be a part of this syndrome [7-9].

In our case the diagnosis of multifocal sclerosing thyroiditis, in according with Armed Forces Institute of Pathology (AFIP) textbook on thyroid tumors, was made only after histological examination. In fact, multifocal sclerosing thyroiditis differs from Riedel's thyroiditis, since the lack of extraglandular extension and oblitering flebytis, considered typical features of Riedel's thyroiditis [6].

Established the massive presence of IgG4 and IgA within sclerosing foci (Figure 4), this clinical case can be part of the new discovered disease, called IgG4-Related Sclerosing Disease [10].

Having regard to the risk of a systemic sclerosing disease, CT scan was performed and IgG4 plasmatic levels were measured, as well as anti-TPO and anti-SCl70 antibodies. Normal levels of these antibodies and no abnormalities showed on CT scan excluded a multiorganic involvement. The contemporary massive presence of IgG4 within the thyroid reveals that the thyroid gland can be the first (or often the only site) target of autoimmune diseases, as occurs for the other forms of thyroiditis. Multifocal sclerosing thyroiditis can be identified as the first stage of multifocal fibrosclerosis [4,9,10].

The presence of nuclear atypia in multifocal sclerosing thyroiditis may engage a differential diagnosis with papillary carcinoma. The negativity of anti HBNE-1 at himmunohistochemical examination is essential to exclude the presence of papillary carcinoma $[4,10]$.

In conclusion, multifocal sclerosing thyroiditis, in according with AFIP textbook on thyroid tumors [6], represents a separate entity from Riedel's thyroditis. We can consider, moreover, the multifocal sclerosing thyroiditis as the first stage of multifocal IgG4-related sclerosing disease for the presence of IgG4 within fibrosclerotic tissue of thyroid.

Once diagnosis is made, total body CT-scan and plamatic IgG4 levels must be investigated in order to exclude or confirm the presence of systemic disease.

\section{REFERENCES}

[1] J. K. Ormond, "Bilateral Ureteral Obstruction Due to Envelopment and Compression by an Inflammatory Retroperitoneal Process,” The Journal of Urology, Vol. 59, No. 6, 1948, pp. 1072-1079. 
[2] N. R. Barrett, "Idiopathic Mediastinal Fibrosis," The British Journal of Surgery, Vol. 46, No. 147, 1958, pp. 207218. doi:10.1002/bjs.18004619703

[3] D. E. Comings and K. B. Skubi, "Familial Multifocal Fibrosclerosis,” Annals of Internal Medicine, Vol. 66, No. 5, 1967, pp. 884-892.

[4] G. Hamed and K. Tsushima, "Inflammatory Lesions of the Lung, Submandibular Gland, Bile Duct and Prostate in a Patient with IgG4-Associated Multifocal Systemic Fibrosclerosis,” Respirology, Vol. 12, No. 3, 2007, pp. 455-457. doi:10.1111/j.1440-1843.2007.01053.x

[5] M. David and M. D. Yousem, "Thyroid and Parathyroid Gland Pathology: Role of Imaging,” Otolaryngologic Clinics of North America, Vol. 28, No. 3, 1995, pp. 621-649.

[6] J. Rosai, "Tumors of the Thyorid Gland," 3rd Series, Armed Forces Institute of Pathology, Washington DC,
1992.

[7] R. D. Lait and S. G. Hubsher, "Sclerosing Cholangitis Associated with Multifocal Fibrosis: A Case Report,” Gut, Vol. 33, No. 10, 1992, pp. 1430-1432. doi:10.1136/gut.33.10.1430

[8] G. W. Aylward and T. J. Sullivan, "Orbital Involvement in Multifocal Fibrosclerosis," The British Journal of Ophtalmology, Vol. 79, No. 3, 1995, pp. 246-249. doi:10.1136/bjo.79.3.246

[9] A. Oezgen and A. Cila, "Riedel's Thyroiditis in Multifocal Fibrosclerosis: CT and MR Imaging Findings,” American Journal of Neuroradiology, Vol. 21, No. 2, 2000, pp. 320-321.

[10] K. Hirabayashi and G. Zamboni, "IgG4-Related Disease,” Pathologica, Vol. 104, No. 2, 2012, pp. 43-55. 